\title{
Subjective representations of educational design systems
}

\author{
Daniel K. Schneider ${ }^{1}$, Kalli Benetos ${ }^{1}$, Jean-Philippe Pernin ${ }^{2}$, Valérie Emin ${ }^{2,3}$ \\ ${ }^{1}$ TECFA, Université de Genève \\ ${ }^{2}$ MeTAH, Laboratoire d'Informatique de Grenoble \\ ${ }^{3}$ EducTice, Institut National de Recherche Pédagogique, Lyon \\ Daniel.Schneider@unige.ch
}

Draft version submitted to VIDLATEL 2009, First International Workshop on

Visual Design Languages and Applications for Technology Enhanced Learning

( http://elearn.pri.univie.ac.at/vidlatel )

in conjunction with IEEE ICALT 2009, July 17, 2009, Riga, Latvia

\begin{abstract}
This paper explores the benefits of repertory grid technique (RGT) for research on educational design systems. We identified several avenues for research, e.g. comparative analysis of families of design languages; global analysis of approaches, languages and systems; needs analysis; identification of constructs that dominate the thinking of various subcommunities. We present a small feasibility study on idiographic representations of design systems at large and conclude with plans for further investigation.
\end{abstract}

\section{Introduction}

Gibbons \& Brewer [3] argue that "Design languages, formal or intuitive, lie at the heart of all design and development processes and tools". Waters \& Gibbons point out that "Notational systems, used in mature fields of study, are closely related to design languages. [...] Instructional technology is one field that can benefit from a notation system enabling designers to duplicate, execute, and communicate their ideas." [12] This suggests that there is a strong connection between design languages and educational modeling languages, as well as between "design" and "communication".

Several authors define dimensions for educational design languages and educational modeling languages that can be used to create taxonomies. E.g. Gibbons and Brewer [3] identified seven dimensions along which design languages may vary and Boot et al. [1] made a list of seven layers that design documents may include.

Such analytical definitions and feature lists are necessary for the creation of a common language for the various stakeholders interested in design languages and related objects. However, we suggest completing such initiatives with the analysis of idiographic representations of design language researchers, developers and interested "users". We hope to identify design issues and use cases that the various communities may not be aware of and that analytical methods cannot identify. In addition, we aim to improve exchange between communities that otherwise do not interact.

\section{Idiographic constructs and repertory grid technique}

Repertory grid technique (RGT) was invented in the 1950's by George Kelly [8] in the framework of this Personal Construct Theory. RGT is based on the assumption that people's view of objects with which they interact is made up of an idiographic (individual) collection of related similarity-difference dimensions, referred to as personal constructs. RGT has been used in a large variety of fundamental and applied research projects and one of its strength is that it allows the elicitation of perceptions without researcher interference or bias.

A common way to describe the RGT is as the identification of a set of elements within a topic (e.g. a set of design languages) which are then rated according to certain criteria termed constructs. Elements and constructs can be defined by the researcher, but are usually elicited from the subject by a so-called triadic method. Participants will first name a few elements with which they are familiar, e.g. names of design systems. They have to compare triads of elements, for instance design A with designs B and C, and then state 
in what aspect two are similar and the third is different. This procedure is repeated with other combinations of elements until no more new constructs are elicited from the user and until all elements can be discriminated in the construct's space.

The output is a grid, which records a subject's ratings, usually on a 5- or 7-point scale, of $m$ elements in terms of $n$ constructs [2]. This resulting grid can then be analyzed with various data analysis techniques, such as visual inspection, factor and cluster analysis. In addition, there exist content analysis methods for analysis across participants.

RGT does not seem to be popular in educational technology research. It has been used quite extensively in HCI, software engineering [11] and management for various purposes, e.g. design of artifacts, knowledge engineering, requirements analysis, representation of information spaces, search engine analysis or human resource management. In education we found a few papers on topics like needs analysis [6], learning materials, evaluation of training programs, personal development, and components of LMS. From looking at this large body of research [8], we identified a few interesting ideas on how to carry out research on educational design systems and languages.

\section{RGT and educational design systems}

RGT, both as a data elicitation and analysis methodology and a research philosophy, could be used for various purposes as our short review above suggests. We outline four of the promising avenues that we intend to follow up in further research.

(1) We may consider that research sub-communities such as visual design language researchers implicitly define a parallel design situation with various competing approaches. Hassenzahl and Wessler [4] argue that user-based RGT-supported evaluation of artifacts in a parallel design situation may produce rich and concrete data to guide design. Such a "similar systems" analysis has shown to be easy to conduct.

(2) Analysis of "most different systems" is more difficult, but allows the gaining of additional insights. Clearly defined design and modeling languages are not the only means to design pedagogical scenarios. Since scenario design concerns a large community we suggest throwing all kinds of design approaches together and seeing what construct systems emerge. Examples of such systems are LAMS (a visual design interface), Compendium (a concept map editor), Pencil \& Paper, structured learning object editors (Reload LD editor), content languages like eLML, and CSCL tools like
Collage and COFFEE. We explored this avenue in the feasibility example below.

(3) After applying RGT to a large diverse population, e.g. researchers from different subcommunities as well as expert users, we could produce a typology of different construct systems that would help to bridge communication between people and to identify essential features for design systems.

(4) RGT can be used to create "pictures" of how users perceive a single system. E.g. instead of comparing aspects of different systems, we could look at tools and features of single systems. E.g. LAMS could be studied by eliciting constructs from active LAMS users regarding the various activity tools.

\section{A feasibility example}

To explore the interest and the feasibility of RGT to study perceptions of a global design "systems" space, we created two repertory grids with the RepGrid IV software, one for the second co-author $\mathrm{KB}$ who considers herself to be somewhat familiar with some systems and one for the first co-author DKS who considers himself to be well informed about design systems.

\section{Procedure}

The two participants agreed to select at least six design systems with varying features from a list [7]. RepGrid IV's built-in triadic elicitation script [10] was used to extract at least four constructs (labeled aspects). In a next step, ratings for each aspect were adjusted. Construct names were also adjusted during this process, being usually made more general. Finally "break match" warnings about non-discriminated elements or non-discriminating constructs were followed up if possible. Participant KB was asked to name a few systems she was vaguely familiar with and all these systems were then shortly demonstrated and sometimes shown again during the elicitation process that lasted about 2 hours. Participant DKS started with KB's list of elements and added his own constructs, initially through a triadic elicitation. He then added elements and constructs by following up on all of RepGrid IV's break match warnings. At the end, both participants adjusted scores by looking at all elements for each construct.

\section{The educational technologist's model (KB)}

The two-way cluster analysis in fig. 1 shows that systems considered fall into very distinct categories. At a (low) $70 \%$ cut-off rate we get 4 types. eLML (a semantic content markup editor) and coUML are 
isolated. We then find a group of production/editing tools around LAMS, and another group including learning design editors. Constructs that cluster together are all-in-one editing / just a representation and made for learners / teachers. Easy/difficult to use is not far from this category. Then, we find linear/non-linear scenarios and editing tool / modeling language in a cluster. Finally scenario design / content editing is really different from all others.

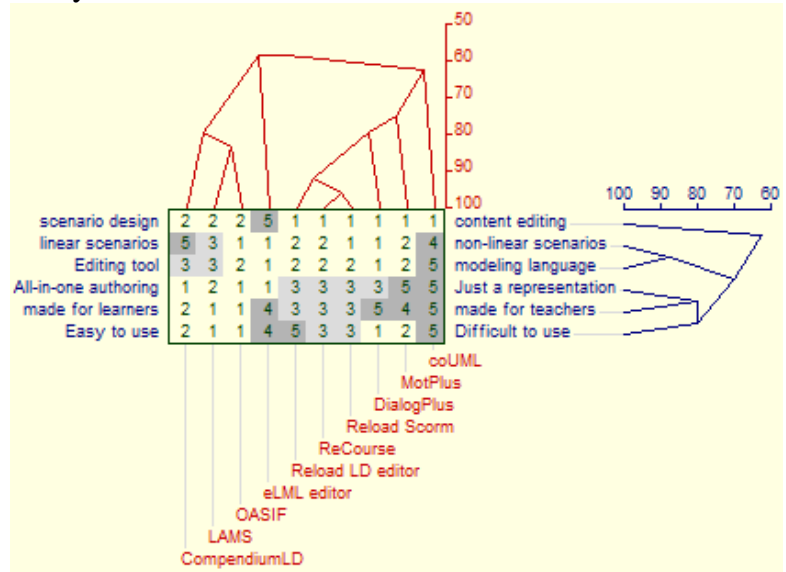

Figure 1: Two way cluster analysis of KB's grid The factor analysis in fig. 2 summarizes the same information with two factors: the first could be named Production orientedness / representation but the second one is more difficult to label. Topical proximity of systems is different since the two factors only explain $67 \%$ of the variance.

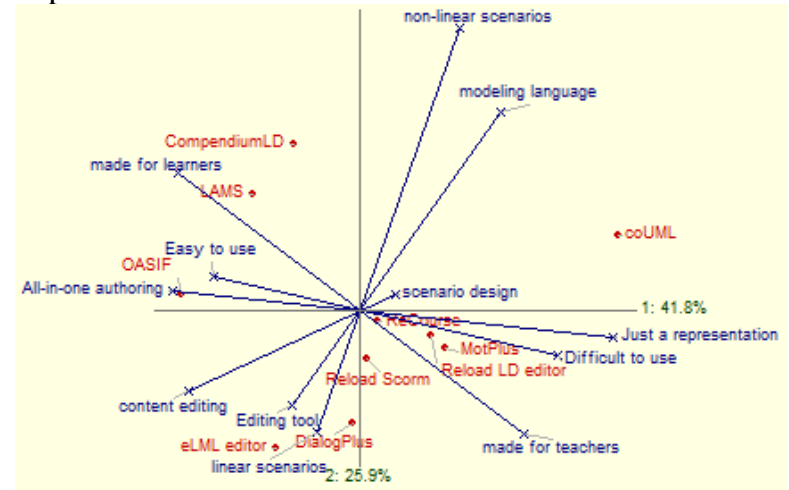

Figure 2: Factor analysis of KB's grid

\section{The expert user's model (DKS)}

The second grid shows a larger variety of clusters and constructs, which is no surprise. It is interesting to notice that he perceived closed source, on-line and complete systems in the same cluster. Being alive and not LD capable was another. High level easy and project-oriented also go together. It is also surprising that systems as different as CeLS and Knowledge Forum and LAMS form a cluster. Or coUML,
Recourse and Reload another. Looking at the two principal components it is easy to see why. The participant's most important emerging factor can be named complete (online) system that allows dynamic planning vs. Design tool, LD capable and standards based. Or more simply fit for real world use. The second factor opposes design languages vs. closed source on-line main-stream systems. Both factors only explain 52\% percent.

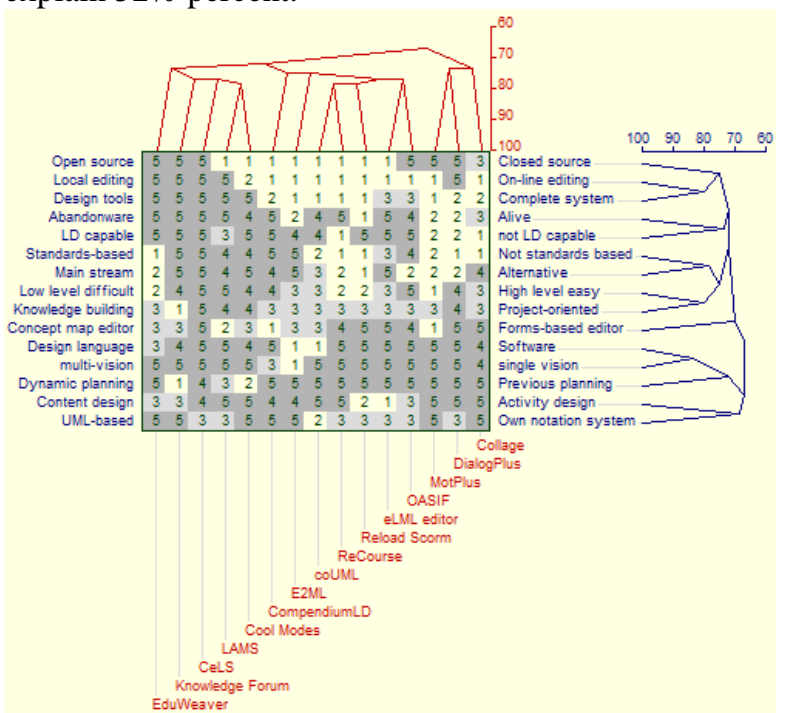

Figure 3: Two way cluster analysis of DKS's grid The $3^{\text {rd }}$ factor (10\% explanation) not shown here could be named activity-based concept map vs. form-based based content editor.

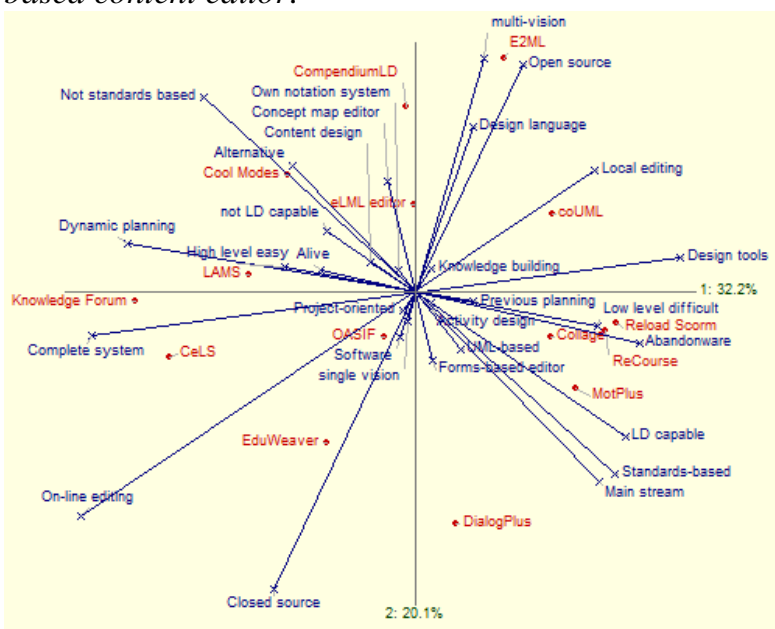

Figure 4: Factor analysis of DKS's grid

\section{Discussion and issues for further work}

The purpose of this study was to explore the potential of RGT for educational design languages research and not to interpret data of just two individuals. We therefore will stick to some methodological issues. 
First of all we notice (and that is no surprise given the two participants) that dominating factors have to with how a system can be used. Without doubt, other user categories will come up with completely different construct systems that would be interesting to contrast and that should enter design and evaluation considerations. Idiographic repertory grids therefore are suitable for highlighting how practical and other use case considerations correlate with perceived characteristics and how various systems fit into the construct space.

The repertory grid technique (RGT) is best used when participants have practical experience with the domain. They must be able to identify representative elements and be able to compare them through a set of (their own) criteria. This is of course a problem with educational design languages. In order to carry out RGT-supported research within a wider population one has to train participants, e.g. in a one-day workshop. In addition, vignettes (e.g. a short web page) describing each system should be created to assist recall.

Elicitation and analysis of DKS's grid revealed several other methodological issues. This $15 \times 15$ grid was elicited by a combination of triadic elicitation and following up break match warnings. Despite removing very similar systems (e.g. Reload LD editor with respect to ReCourse), the participant felt that more systems and constructs should have been added. However, a 15x15 grid already is quite complex and difficult to interpret and should reveal enough data.

Therefore, we should decide on a replicable method that will insure that the most important constructs will enter the grid. The RepGrid software elicitation script will point out similar systems that need to be differentiated by a construct. Instead of introducing a new construct judged to be of little concern, we should remove the system that is less known. We found the trialogic procedure with randomly selected systems useful since it seems to help finding "natural" and strong constructs. However, we also suggest halting this procedure in the middle of the process. Participants will be shown results (e.g. the two-way cluster analysis) and if they find that two elements are too close, a construct that discriminates the two should be added. If two constructs are judged to be too close, we may elicit a system that differentiates the two.

Finally, this leads us to the discussion of whether comparing very different systems is a good strategy since construct spaces quickly become complex. Data reduction will not produce "tight" clusters and variance explained by the two first factors will be below 50\%. Such data is still very useful to the educational design researcher but such an analysis should be completed by most similar systems designs in order to identify more focused constructs about a class of design systems.

We did not explore analysis across construct systems. Before doing so, we have to interview a larger set of participants and then engage in content analysis and laddering (in-depth exploration of constructs) for grouping the most commonly used constructs. A sample size of about 15-25 persons should generate enough constructs that are representative of the universe of meaning [2]. Another avenue is to explore group elicitation techniques [9], where common grids for expert subgroups are developed in a two-phased approach.

Finally, we suggest comparing idiographic constructs with constructs obtained through a fixed nomothetic grid. The constructs could be assembled from a synthesis of analytical and evaluation grids found in the literature. The elements should be a representative selection of various and somewhat known design systems.

In conclusion of this little exploration we put forward the conjecture that RGT could become a useful communication tool among design researchers and between researchers and expert users.

\section{References}

1. Boot, E.W., J. Nelson, J. J.G. van Merriënboer \& A. S. Gibbons. "Stratification, elaboration and formalisation of design documents", British Journal of Educational Technology 38 (5), 2007, pp. 917-933.

2. Dillon, A. Designing Usable Electronic Text, CRC, 1994.

3. Gibbons, A. S. and E.K. Brewer, "Elementary principles of design languages" and design notation systems for instructional design". In J.M. Spector, et al. (eds.), Innovations to instructional technology, Erlbaum, 2005 pp. 111-129.

4. Hassenzahl, M. \& Wessler, R., “Capturing design space from a user perspective: the Repertory Grid Technique revisited”. International Journal of Human-Computer Interaction, 2000, 12 (3/4), 2000, pp. 441-459.

5. Kelly G, The Psychology of Personal Constructs, New York: Norton, 1955.

6. Peters, W.L. "Repertory Grid as a Tool for Training Needs Analysis”, The Learning Organization, 1 (2), 1994, pp. 23-28.

7. Schneider, D, Educational design language, wiki page, http://edutechwiki.unige.ch/, retrieved 23/2 2009.

8. Schneider, D, Repertory grid technique, wiki page, http://edutechwiki.unige.ch/, retrieved 23/2 2009.

9. Shaw, M.L.G \& B.R Gaines. "Comparing Conceptual Structures: Consensus, Conflict, Correspondence and Contrast”, Knowledge Acquisition 1(4), 1989, pp. 341363. 
10. Shaw, M.L.G.. On Becoming A Personal Scientist: Interactive Computer Elicitation of Personal Models Of The World. London, Academic Press, 1980.

11. Tan, F.B., Hunter, M.G., "The repertory grid technique: a method for the study of cognition in information systems”, MIS Quarterly, 26 (1), 2002, pp.3957.

12. Waters, S., H. \& A. S. Gibbons, "Design languages, notation systems, and instructional technology: A case study”, Educational Technology Research and Development, 52(2), 2004, pp. 57-69. 